\title{
Obesity, Non-Alcoholic Fatty Liver Disease and Eating Behavior Disorders
}

\author{
Habiba Libaton*1, M Mziwira ${ }^{1,2}$ and R Belahsen ${ }^{1}$ \\ ${ }^{1}$ Training \& Research Unit on Nutrition and Food Sciences, Chouaib Doukkali University, Morocco \\ ${ }^{2}$ Normal Higher School- Hassan II University, Life Sciences, Morocco \\ *Corresponding author: Habiba Libaton, Training \& Research Unit on Nutrition \& Food Sciences, Chouaib Doukkali University, El \\ Jadida, Morocco.
}

To Cite This Article: Habiba Libaton. Obesity, Non-Alcoholic Fatty Liver Disease and Eating Behavior Disorders. Am J Biomed Sci \& Res. 2019 4(2). AJBSR.MS.ID.000775. DOI: 10.34297/AJBSR.2019.04.000775

Received: June 26, 2019 | Published: July 23, 2019

\section{Opinion}

Non-alcoholic fatty liver disease related to obesity is a growing epidemic worldwide, resulting in the accumulation of triglycerides and free fatty acids in the liver. In Morocco, the increase in non-alcoholic fatty liver disease related to obesity highlights the contribution of environmental factors to this public health problem, without denying the caloric contribution that is due to macronutrients, high energy density and low oxidation. In fact, the dietary transition experienced by Morocco following the production of the food industry and the presence of the media has turned the Mediterranean dietary model characterized by a diet rich in starch and fiber, low in fat and a physically active life to a pattern with more diversified diet rich in sugars, saturated animal fats and processed foods, low in fruits, vegetables and fibre and a sedentary and stressful lifestyle [1].

In addition, palatability, variety, speed of ingestion and availability of food make obesity, metabolic disorders and nonalcoholic fatty liver disease to increase in Moroccan society. Indeed, in children under 5 years, $12.5 \%$ are overweight and $2.6 \%$ are obese). For adults over $18,20 \%$ are obese, $29.3 \%$ are hypertensive, $10.6 \%$ are diabetic and $10.5 \%$ have hypercholesterolemia. In addition, non-communicable diseases account for $80 \%$ of all deaths (WHO, 2018). In the face of obesity and its co-morbidities, particularly non-alcoholic fatty liver disease, progressive weight loss is recommended. However, the strong restriction of food intake for the purpose of weight control induces certain counterregulatory behaviors. Namely, the over-consumption that arises from the difficulty to adjusting consumption according to food energy content or, in response to the emotions or in the presence of environment stimuli (meals, the family environment). The alternation between over-consumption and the strong restriction constitutes eating disorders leading to psychosocial, metabolic and cardiovascular complications that can lead to death [2].

\section{Purpose}

The purpose of this study is to prevent behavioral eating disorders and their consequences on health.

\section{Objective}

The objective of this work is to study the prevalence of eating behavioral disorders in obese people who have non- alcoholic steatosis through a retrospective study, dealing with patient records at the endocrinology at Med VI Medical Center in Marrakech. The data will be analyzed and discussed in association with variables the eating behavior disorders, body mass index et NAFLD.

The question that arise is: what is the pathophysiology of eating behavior? How to overcome the consequences of these disorders on non-alcoholic fatty liver disease and obesity?

Keywords: Non-Alcoholic Fatty Liver Disease; Obesity; Eating Behavior Disorders

\section{References}

1. Chamberlain SR, A Redden S, Grant JE (2017) Calorie intake and gambling: Is fat and sugar consumption « impulsive »? J Gamb Stud 33(3): 783-793.

2. Iceta S, Julien B, Seyssel K, Laville M, Disse E (2018) Ghrelin concentration as an indicator of eating-disorder risk in obese women. Diabetes Metab S1262-S3636(18): 30008-30009. 